Planetary Systems in the Universe - Observation, Formation and Evolution

Proceedings IAU Symposium No. 202, (C)2004 IAU

Alan Penny, Pawel Artymowicz, Anne-Marie Lagrange, 8 Sara Russell, eds.

\title{
Protoplanetary disc evolution in magnetically layered disc models
}

\author{
Philip J. Armitage \\ Max-Planck Institut für Astrophysik, Karl-Schwarzschild-Str. 1, \\ Garching D85741, Germany
}

\begin{abstract}
I discuss protoplanetary disc evolution under the assumption that magnetohydrodynamic turbulence and self-gravity are the sole sources of angular momentum transport. This assumption implies a magnetically layered disc structure which leads to unsteady accretion, and larger disc masses at late epochs. The resulting environment for planet formation and migration differs qualitatively from the highly simplified - almost toy - models often adopted.
\end{abstract}

\section{Introduction}

Protoplanetary discs evolve substantially over their lifetime. In addition to a decline in the disc mass as material accretes (Hartmann et al. 1998), there are violent outbursts - FU Orionis events - during which the accretion rate through at least the inner disc can be as high as $10^{-4} M_{\odot} \mathrm{yr}^{-1}$. Given these observed complexities, the static or steadily evolving nebula models considered by planet formation theorists are obviously a poor approximation. Of course it is hard to do better, not least because current observations provide few constraints at the critical radii for planet formation of $r \sim 1$ AU. Here, I discuss some of the implications for planet formation of one recent theoretical model for protoplanetary disc evolution (Gammie 1996) which, although itself uncertain, attempts to include 'best guess' estimates of the important physical processes.

\section{Magnetically layered disc models}

Turbulence driven by magnetic instabilities is the best understood source of viscosity in accretion discs (Balbus \& Hawley 1991). As pointed out by Gammie (1996), this has important implications for protoplanetary disc structure, because at the low temperatures $\left(T \sim 10^{2} \mathrm{~K}\right)$ found at $r \sim 1 \mathrm{AU}$, the disc is so weakly ionized that MHD turbulence is suppressed. If there are no other pervasive sources of angular momentum transport, the disc is expected to develop a novel structure comprising three radial regions:

(1) An inner region at $r \lesssim 0.1 \mathrm{AU}$ hot enough that MHD turbulence is an efficient source of viscosity. Here, 'hot enough' means a temperature of $T_{\text {crit }} \approx 10^{3} \mathrm{~K}$, above which ionization of the alkali metals leads to a sharp jump in the ionization fraction of the disc.

(2) A magnetically layered region at intermediate radii $(r \sim 1 \mathrm{AU})$ where MHD turbulence near the disc midplane is suppressed due to the low ion- 

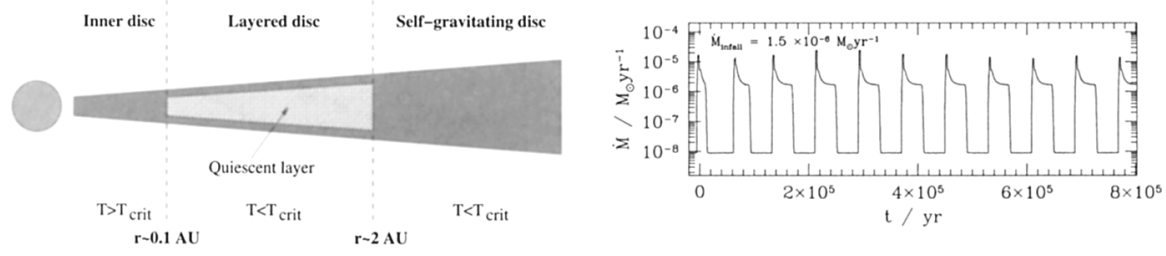

Figure 1. The magnetically layered disc model, illustrated in the left panel, creates a bottleneck to accretion at radii $r \sim 1 \mathrm{AU}$. This leads (right panel) to episodic accretion for a wide range of mass fluxes through the outer disc.

ization fraction. A trickle of accretion may persist through a surface layer ionized by cosmic rays, but at a much reduced rate $\dot{M} \sim 10^{-8} M_{\odot} \mathrm{yr}^{-1}$.

(3) An outer region where angular momentum is transported by self-gravity, or by MHD turbulence where the surface density is low enough that cosmic rays can ionize the whole thickness of the disc.

This structure is illustrated schematically in Figure 1.

We have recently calculated the evolution of such a layered disc model using a one-dimensional (radius only) model (Armitage, Livio \& Pringle 2001). The model uses a one zone treatment of the vertical structure, and includes simplified $\alpha$ prescriptions to describe angular momentum transport from both MHD turbulence and disc self-gravity.

Episodic accretion is an immediate consequence of the model (Gammie 1996, 1999; Armitage et al. 2001). If the accretion rate through the outer disc exceeds the maximum that the magnetically layered region can support, the disc will be unable to reach a steady state. Matter will then pile up in the quiescent layer. This causes the self-gravitating region of the disc to encroach into the layered region, heating it up and eventually restarting MHD turbulence. A cycle is obtained in which angular momentum transport in the disc is alternately dominated by MHD turbulence and self-gravity. Typical outburst accretion rates are $\dot{M} \sim 10^{-5} M_{\odot} \mathrm{yr}^{-1}$. The timescales for the outbursts depend upon the details of the model. In our calculations, the outbursts are long $\left(\sim 10^{4} \mathrm{yr}\right)$, and the most likely observational manifestation is modulation of the rate of outflow from the inner disc. It has also been suggested that a shorter version of this cycle could lead to FU Orionis events (Gammie 1996, 1999).

\section{Implications for planet formation and migration}

Layered disc models imply a different environment for planet formation and migration than most other disc models. Notable features include:

(1) For most of the time, the gas near the disc midplane at $r \sim 1 \mathrm{AU}$ is not turbulent. Dust may be able to settle until it begins to generate its own turbulence (Weidenschilling \& Cuzzi 1993).

(2) The layered region of the disc creates a bottleneck through which accretion is restricted. Therefore (a) the disc mass is much higher $\left(\sim 0.1 M_{\odot}\right)$ at 
late epochs, and (b) the outer disc remains self-gravitating, or nearly so, even at $t \sim 1 \mathrm{Myr}$. Note that most of the 'additional' mass is locked up at small radii where the disc is highly optically thick, so this prediction does not instantly fall foul of $\mathrm{mm}$-wavelength estimates of the disc mass.

(3) The disc within a few AU of the star is subject to repeated, rapid, episodes of heating during outbursts of accretion.

(4) The migration rate for Earth mass bodies (Ward 1997) is reduced at the outer boundary of the layered region, since the greater dissipation at larger radii leads to a flat or increasing central temperature with radius.

(5) Planets embedded in gaps encounter an almost inviscid disc in the magnetically layered region. Migration will be slow except during outbursts.

(6) The low viscosity in the disc may allow growth of the eccentricity of massive planet orbits.

Of course the layered model is subject to substantial uncertainties. The most serious challenge to the basic concept is the suggestion that purely hydrodynamic instabilities in protoplanetary discs can lead to significant angular momentum transport (Klahr \& Bodenheimer, this meeting). Even absent such instabilities, the current models are highly simplified. Particularly lamentable is the lack of a self-consistent treatment of stellar irradiation, which is more important for layered discs than for fully viscous models due to the reduced viscous dissipation in the layered region. Future work aims to address these shortcomings.

Acknowledgments. Thanks to my collaborators on this work, Jim Pringle and Mario Livio, and to the organisers for a stimulating meeting.

\section{References}

Armitage, P. J., Livio, M., \& Pringle, J. E. 2001, MNRAS, 324, 705

Balbus, S. A., Hawley, J. F. 1991, ApJ, 376, 214

Gammie, C. F. 1996, ApJ, 457, 355

Gammie, C.F. 1999, in ASP Conf. Series 160, Astrophysical Disks, ed. J. A. Sellwood \& J. Goodman (San Fransisco: ASP), 122

Hartmann, L., Calvet, N., Gullbring, E., \& D'Alessio, P. 1998, ApJ, 495, 385

Ward, W. R. 1997, Icarus, 126, 261

Weidenschilling, S. J., Cuzzi, J. N. 1993, in Protostars and Planets III, ed. E. Levy \& J. I. Lunine (Tuscon: Univ. Arizona Press), 1031 